\title{
CORROSION INHIBITION OF HEAT TREATED MILD STEEL WITH NEEM LEAVE EXTRACT IN A CHLORIDE MEDIUM
}

\author{
Tolumoye J. Tuaweri ${ }^{1}$, Ezenwa A. Ogbonnaya ${ }^{2}$, Okechukwu O. Onyemaobi ${ }^{3}$
}

${ }^{I}$ Department of Mechanical/Marine Engineering, Niger Delta University, Wilberforce Island, Bayelsa State, Nigeria
${ }^{2}$ Department of Mechanical/Marine Engineering, Niger Delta University, Wilberforce Island, Bayelsa State, Nigeria
${ }^{3}$ Department of Mechanical/Marine Engineering, Niger Delta University, Wilberforce Island, Bayelsa State, Nigeria

\begin{abstract}
Influence of Neem leave extract on the corrosion inhibition of mild steel in a chloride medium was investigated. It was carried out by immersing as-received and heat treated mild steel coupons in salt water with various concentrations of Neem leave extract ranging from 0-20\%. Method of investigation was gravimetric, based on weight loss of coupons in the different solutions. The results show that mild steel samples immersed in salt water without Neem leave extract exhibited a much higher weight loss as compared to those with Neem leave extract. A unique trend observed in these investigations was that weight loss was higher for the as-received than the heat treated samples prior to addition of Neem leave extract into the corrosive medium. On addition of the Neem leave extract, a reversed trend was observed where weight loss of the heat treated became higher than the as-received coupons. Change in corrosion rate was insignificant for the control samples for both as-received and heat treated samples. On the contrary, an evident decreasing trend was observed for the heat treated samples while those of as-received were insignificant with time. However. 5\% inhibitor showed best corrosion inhibition with the least corrosion rates. The overall results indicate that Neem leave extract effectively inhibits mild steel corrosion in a chloride medium
\end{abstract}

Keywords: Corrosion, mild steel, salt water, inhibitor, Neem leave, heat treatment

\section{INTRODUCTION}

Mild steel, referred to as low carbon steel is widely used in industries because it is relatively inexpensive to produce and has good ductility, toughness, weldability and machinability; hence it can be used in different applications [1]. Typical applications include automobile body components, ships, pipelines, structural shapes, buildings, bridges, railway lines and tin cans, some pots and pans used for cooking etc.[2].

In most cases its serviceability depends on the fact following the initial corrosion attack and its application. Protective films formed tend to reduce the corrosion rate to an acceptable level. However, the conditions and environment which metals operate are many and vary. Despite these varied conditions, steel is expected to function effectively, so there is need to introduce inhibitors in these different conditions and environments that metals operate to help reduce or prevent its corrosion in them, so as to achieve desired results [3].

One of the most practical methods for protection against metallic corrosion is the use of corrosion inhibitors. When small concentrations are added to corrosive media, they either decrease or prevent the reaction of the metal with the media. Inhibitors hinder corrosion reactions either by reducing the probability of corrosion occurrence or by reducing the rate of attack or by both [4]. Two types of inhibitors popularly utilized are the organic and inorganic inhibitors. Although, inorganic substances such as phosphates, chromates, dichromate and arsenates have been found to be effective as inhibitors, they suffer a major disadvantage of higher toxicity and as such their use has come under severe criticism. Consequently, organic substances have become more attractive as anticorrosion agents as these are more eco- friendly and harmless.

Organic substances containing polar functions with oxygen, nitrogen and/or sulphur atoms in a conjugate system have been reported to exhibit good inhibiting properties [5]. To this end, the use of organic substances to inhibit corrosion of metals is well established [6]. However, development of new corrosion inhibitors which effectively inhibit corrosion under a variety of conditions while being environmentally safe and acceptable is a challenging task [7]. Leading in research for such mutuality in both effective inhibition and eco-friendliness are plant extracts. Plant extracts have become important because they are environmentally acceptable, inexpensive, readily available and renewable sources of materials, and ecologically acceptable [8]. Neem tree is an economic tree mostly found in the African rain forest and has high medicinal value. Oftentimes, only small quantities of the extract are utilized for purposes of traditional medicine and the rest disposed off as waste water which could be recycled as a corrosion inhibitor. Although, the use of Neem leave extract as a corrosion inhibitor for carbon steel in $\mathrm{HCl}$ has been reported [9], not a lot is reported on its inhibition of heat treated carbon steel. This study therefore seeks to determine the effect of Neem leaf extract as an organic inhibitor on heat treated mild steel corrosion in salt water. 


\section{MATERIALS AND METHODS}

All investigations were carried out using mild steel coupons; as received and heat treated. Salt water was utilized as the corrosive medium. The range of inhibitor concentrations investigated was between 5-20vol.\%. All investigations were carried in plastic bowls and rubber strings were used to suspend the coupons in each solution. For purposes of minimal error, air tight electron balance was used to weigh the coupons before and after each experiment. Table 2.1 below shows the elemental composition of the mild steel coupons utilized.

Table 2.1 Elemental composition of Mild Steel

\begin{tabular}{|l|ccccccccc|}
\hline Element & $\mathbf{C}$ & $\mathbf{S i}$ & $\mathbf{M n}$ & $\mathbf{P}$ & $\mathbf{S}$ & $\mathbf{C u}$ & $\mathbf{C r}$ & $\mathbf{S n}$ & $\mathbf{F e}$ \\
\hline Wt \% & 0.18 & 0.24 & 0.50 & 0.03 & 0.04 & 0.20 & 0.10 & 0.02 & 98.7 \\
\hline
\end{tabular}

\subsection{Sample Surface Preparation}

Surface finishing was carried out using grade 600 grit paper. Specimen were rinsed in water, degreased in acetone, air dried and weighed immediately to avoid reoxidation before immersion in the test solution. All cut and sheared edges were machined to prevent them from becoming sites of preferential attack. Degreasing was done to remove any dirts, oil or grease that might interfere with the investigations. The volume of test solution was relatively large (a solution volume-to-specimen area of $30 \mathrm{ml} / \mathrm{cm}^{2}$ ) enough to avoid any appreciable changes in corrosivity during the test, either through exhaustion of corrosive constituents or accumulation of corrosion products. The method of supporting the specimens ensured the insulation of the specimen from each other physically and electrically, and also from the plastic container used

\subsection{Inhibitor Preparation}

The Neem leaves were collected from Swali Yenagoa, Bayelsa State, while the sea water was collected from Mgbuosimini at Eagle Island, Rivers State both in Nigeria.

About $410 \mathrm{~g}$ of the leaves was ground using mortar and pestle, added to $1000 \mathrm{ml}$ of water and mixed to enable extraction of the green content. The leaves were well squeezed and filtered twice after mixing so as to have fine extracts without traces of leaf particles. Some active ingredients in Neem leaf extract includes; Azadirachtin, Gedunin and Nimbidin.

\subsection{Test Solution Preparation}

Salt water was poured into a $500 \mathrm{ml}$ beaker containing $0 \%$ inhibitor and then transferred to a plastic bowl were the immersion of the mild steel was done (control sample). Salt water was then poured into $500 \mathrm{ml}$ beakers containing $5 \%$, $10 \%, 15 \%, 20 \%$ respectively of the inhibitor having same molar concentration of $0.41 \mathrm{~g} / \mathrm{mol}$ and poured into different transparent plastic bowls. The process was carried out with two plastic bowls for each percentage considered so as to account for both as- received and heat treated mild steels coupons.

\subsection{Corrosion Study.}

The corrosion rate, $\mathrm{CR}$ in $\mathrm{mm} / \mathrm{yr}$. is given by

$$
\mathrm{CR}=\frac{\mathrm{K} \times \mathrm{W}}{\mathrm{A} \times \mathrm{T} \times \mathrm{D}}
$$

Where,

$\mathrm{W}=$ Mass loss $(\mathrm{g})$

$\mathrm{K}=$ Constant $=8.76 \times 10^{4}$

$\mathrm{A}=$ area of coupon $\left(\mathrm{cm}^{2}\right)$

$\mathrm{D}=$ density of mild steel $\left(\mathrm{g} / \mathrm{cm}^{3}\right)=7.86 \mathrm{~g} / \mathrm{cm}^{3}$

$\mathrm{T}=$ Time of exposure in hours

\section{RESULTS AND DISCUSSION}

\subsection{Effect of Time on Weight Loss}

Weight loss as a function of time is shown in Figs 1-4. From all four figures it is evident that weight loss increased with time. However, the magnitude of increase was not the same for the control bath without inhibitor and those with inhibitor. As evident from Fig. 1, the magnitude of corrosion was directly proportional to time for the baths without inhibitor. Similarly, the bath with 5\% inhibitor also exhibited some degree of proportionality between the weight loss and time. But those with $10 \%$ and $20 \%$ inhibitor exhibited a general tendency to reach a peak and then normalized for the range of durations investigated. This disparity in corrosion behavior between the control sample and those with inhibitors is understandable, as the inhibitor may have conferred some degree of coverage and protection to the surface of the coupons from the corrosive attack of chloride ions in the corrosive medium. When metals are placed in a corrosive medium, the residual charge in the surface of the metal greatly influences the degree of adsorption of the inhibitor and hence the corrosion inhibition efficiency of the inhibitors [10]. Also, Fig. 1 shows an evident difference in the weight loss between the as-received and the heat-treated samples. The as-received sample exhibited a higher weight loss than the heat-treated. Amongst several others, heat treatment is often carried out in order to; relief internal stresses developed during cold working, welding, casting, forging etc; and increase heat, wear and corrosion resistance [11] 


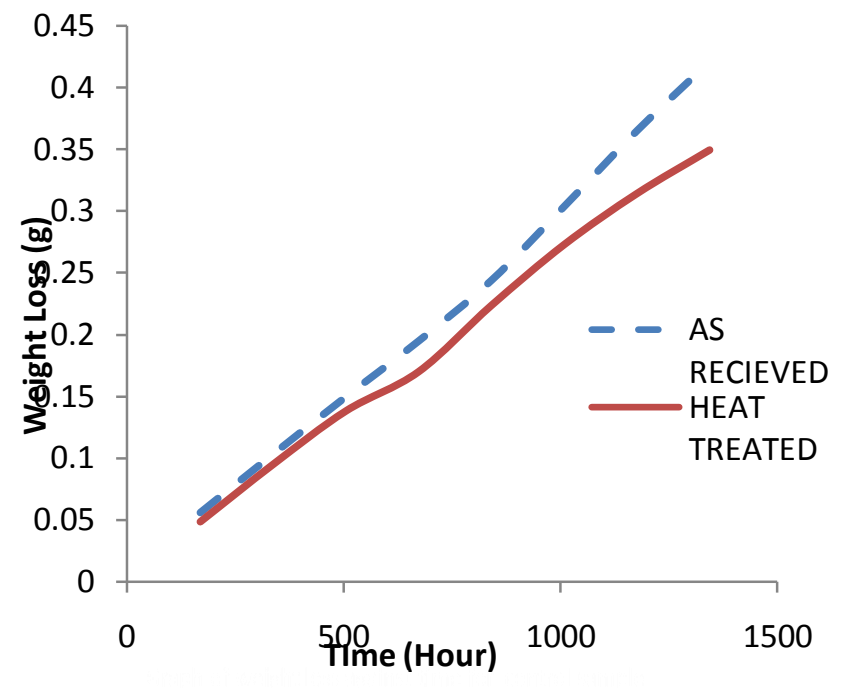

Fig. 1 Weight loss Vs Time for Control Sample

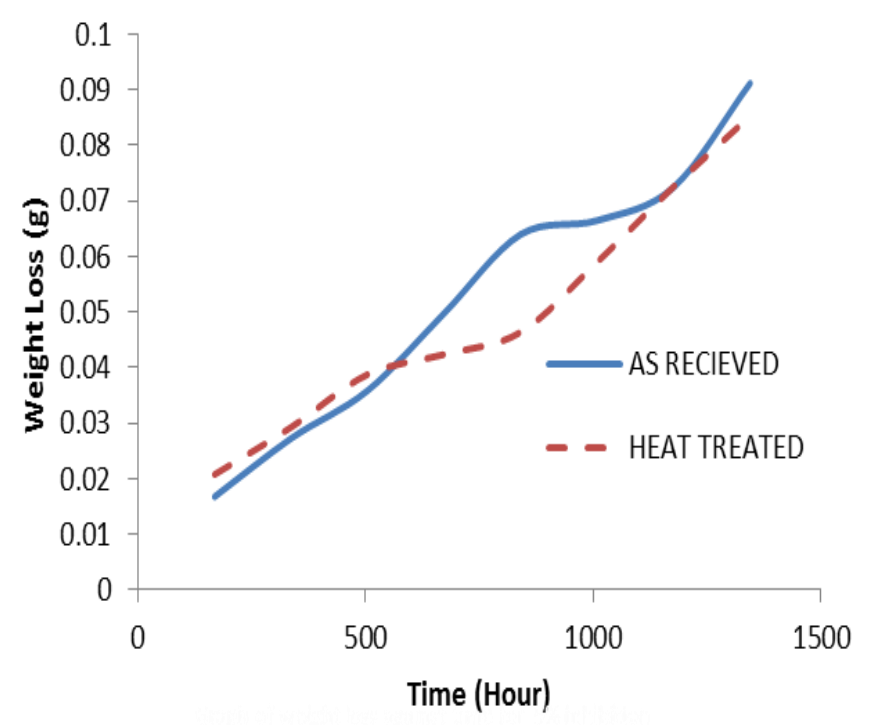

Fig. 2 Weight loss vs time in $5 \%$ inhibitor

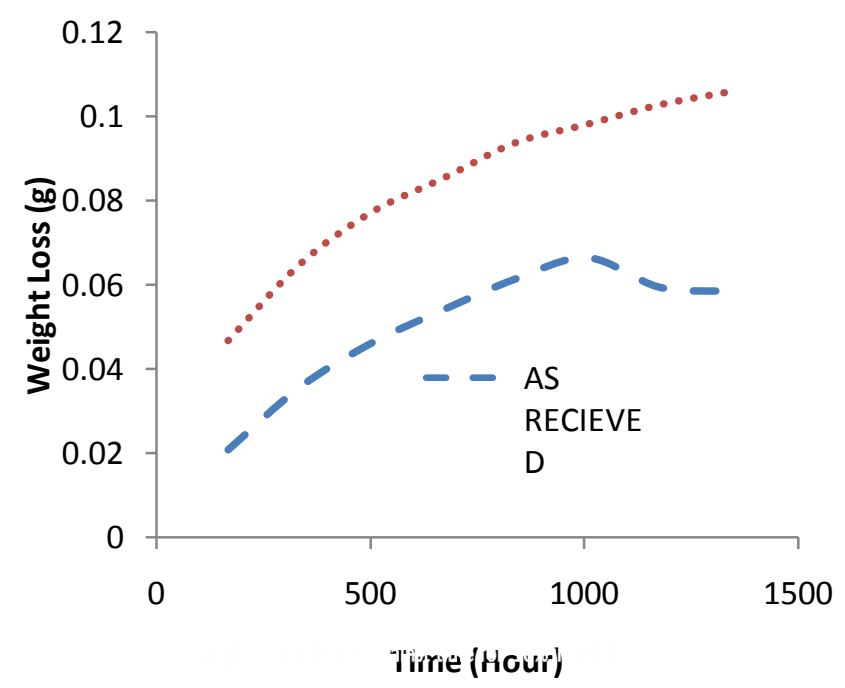

Fig 3. Weight loss vs time for sample in the corrosive medium with $10 \%$ of inhibitor

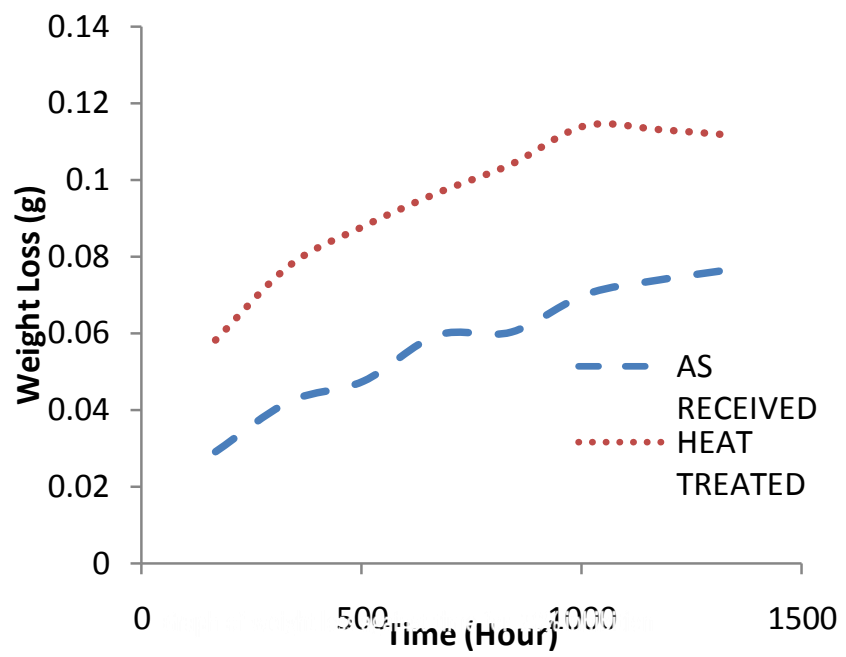

Fig. 4 Weight loss vs time for sample in the corrosive medium with $20 \%$ of inhibitor

\subsection{Effect of Inhibitor on Weight Loss}

Figs 1- 4 show the corrosion resistance behavior of the asreceived and heat-treated samples with $0 \%, 5 \%, 10 \%$ and $20 \%$ of the inhibitor in the corrosive medium. The results show that weight loss of the coupons was much higher for the as-received coupons than those of the heat-treated with $0 \%$ inhibitor in Fig. 1. For Fig 2, a bath with 5\% inhibitor, there was no distinct difference in trend between the asreceived and the heat-treated. However, For Figs 3 and 4 with $10 \%$ and $20 \%$ inhibitor concentrations respectively, there was a distinct difference in the magnitude of weight losses between the as-received and the heat-treated. The trend indicates a gradual change of position between the asreceived and the heat treated coupons with $5 \%$ inhibitor being the point of transition where weight loss of both types of coupons normalizes and then interchanges position with further increase in inhibitor concentration. At $0 \%$ inhibitor, heat-treated samples exhibits better corrosion resistance than the as-received but shows a reverse trend at higher inhibitor concentrations. Reasons for such behavior are not very clear. However, this may not be unconnected with the nature of interactions between the inhibitor molecules and the two different coupons due to possible disparities in their microstructures. Quite significantly too, it is obvious from Figs 2-4 that with the addition of inhibitor all weight loss values were less than $0.12 \mathrm{~g}$ in contrast to Fig 1 where the weight loss values were much higher. This is also a clear indication of the effective inhibitive action of Neem leaf extract on mild steel corrosion. Chances of the heat treated coupons to possess a martensitic microstructure are higher due the fast cooling process the mild steel coupons were subjected to than that of the as-received coupons. During heat treatment of steel, the type of microstructure obtained is largely a function of the cooling rate. A number of phase transformations are possible namely; pearlite, bainite and martensite. Martensite phase is formed when quenching occurs quickly. Its formation is independent of time and tends to have a passive film that is relatively stable when compared to that of as-received coupons which was not heat treated. Persistence of this passive film may have further 
stifled the surface of the heat treated samples and hence become more resistant to corrosive attacks than the asreceived in the absence of inhibitor. Passive and more stable film on the martensitic phase has been reported to be responsible for preventing optimal adsorption of the inhibitor [12]. Pearlite samples in the absence of inhibitors exhibited less corrosion than martensite ones, which was because of more protective layers [13]. In such cases, the martensite phase may have possessed some degree of porosity thereby exposing a larger surface area for corrosive attacks. Generally, corrosion inhibitors confer protection to surfaces via preferential adsorption onto the surfaces or interfaces in a system and alter the surface and interfacial free energies even at low concentrations [14]. Microstructural differences could obviously present differences in surface energies. Since microstructural changes often accompany heat treatment processes, the microstructures of as-received and heat treated mild steel may be different and hence their surface energies. Such differences in surface energies could determine the nature and rates of adsorption of the inhibitor molecules thereby providing unequal protection to both surfaces. Furthermore, presence of martensite in a quenched steel, while greatly increasing its hardness and tensile strength, causes the material to be brittle. Such behavior is hardly surprising, since the formation of martensite is accompanied by severe matrix distortions [15]. In view of the aforementioned, a number of reasons were probably responsible for the higher weight losses and corrosion rates for the heat treated coupon; (1) The more stable passive film on the martensite due to heat treatment probably prevented the adsorption of the inhibitors; (2) Although relatively stable, such protective passive films may have short-lived at specific concentrations of the corrodent thereby exposing more metal surface to further aggressive attacks; (3) The martensite-free microstructure of the as-received mild steel may have been more receptive to the inhibitor molecules enabling them to form a protective inhibitor layer

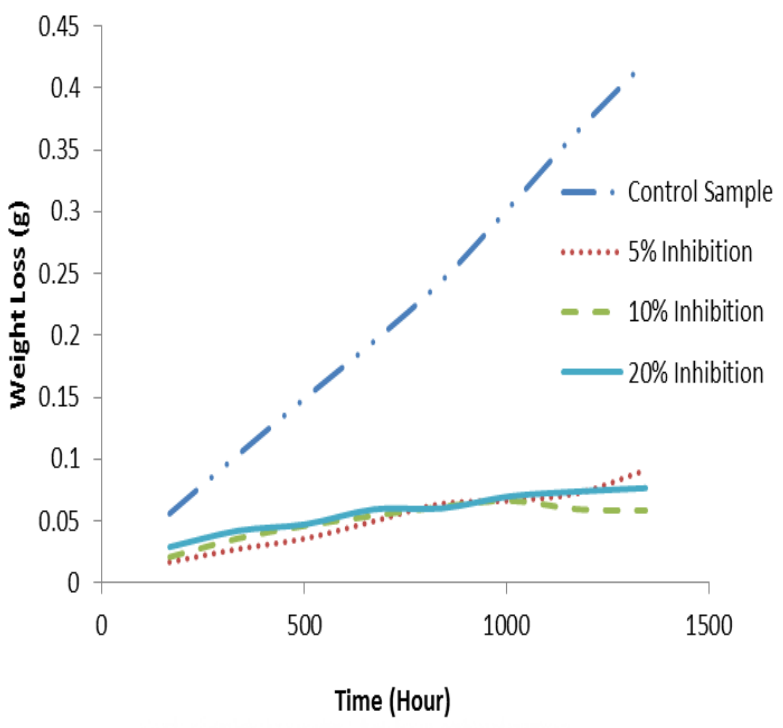

Fig.5 Weight loss vs time for as-received coupons with different inhibitor concentrations

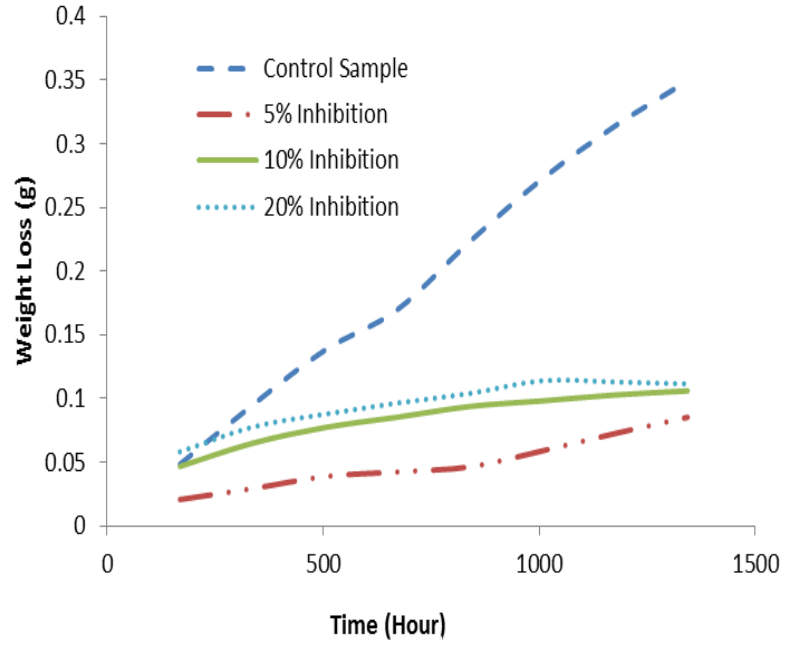

Fig. 6 Weight loss vs time for heat-treated coupons.

\subsection{Effect of Inhibitor Concentration on Weight}

\section{Loss as a Function of Time}

Figs 5 and 6 show the relationship between inhibitor concentration and weight loss as a function of time. It is evident from each of these figures that weight loss increased with time for all the concentrations investigated. On each figure, the margin of weight loss between the inhibited and the uninhibited (control sample) was quite wide indicating that Neem leaf extract effectively inhibited mild steel corrosion. Also, it is evident from both figures that for all inhibited samples, the magnitude of weight loss was higher for the heat treated coupons as compared to the as-received; as most of the weight loss results for the heat treated samples were higher than $0.05 \mathrm{~g}$ (see fig. 6) while those of as-received were slightly lower (see fig. 5). Further attesting to the fact that Neem leaf extract inhibits as-received mild steel better than heat treated. Furthermore, all figures show that corrosion inhibition was better at a concentration of $5 \%$ inhibitor. At higher concentrations, the inhibition was lower progressively with increasing concentrations.

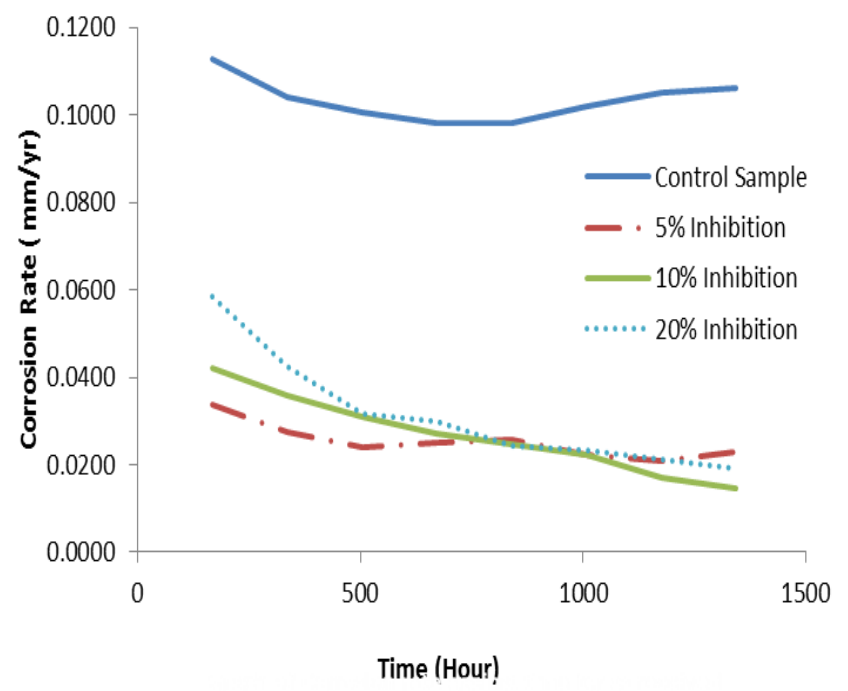

Fig. 7 Corrosion rate vs time for as-received coupons 


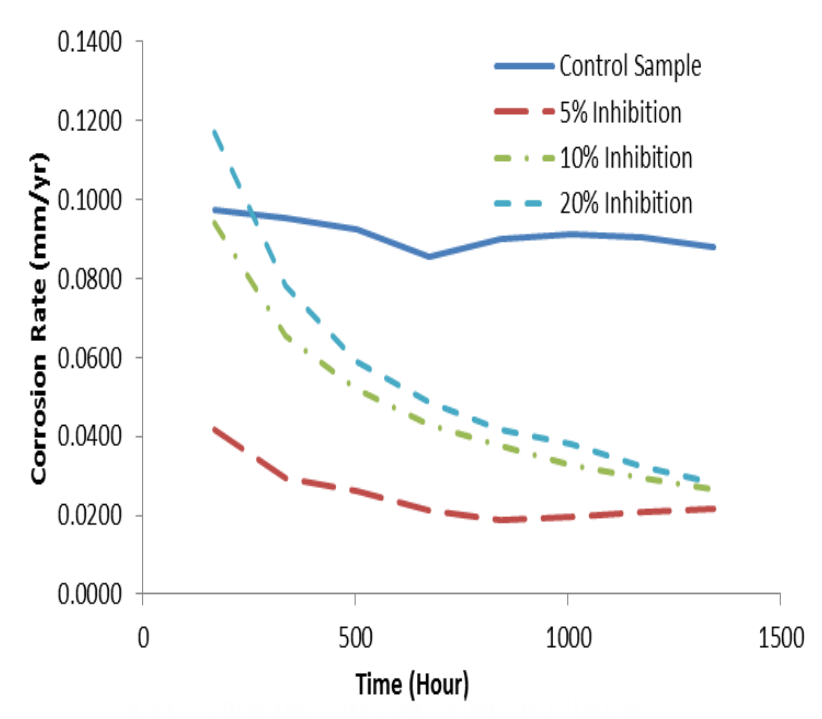

Fig. 8 Corrosion rate vs time for heat-treated coupons

\subsection{Corrosion Rate as a Function of Time}

Figs 7 and 8 show the relationship between corrosion rates, time and inhibitor concentration for the as-received and heat treatment coupons respectively. A general trend in both figs shows that the rate of corrosion gradually decreased with time for all inhibited samples as compared to that of the control sample which exhibited higher but relatively constant corrosion rates. In relation to inhibitor concentrations, the corrosion rates decreased with decreasing inhibitor concentrations. While this range was not very wide for the as-received samples, it took the following decreasing order for the heat treated samples; $20 \%, 10 \%$ and $5 \%$ of inhibitor concentration. For the control samples (as-received and heat treated) on both figs, it is evident that there was no significant decrease in the corrosion rates with time. This is probably due to instability of corrosion products on the mild steel surface providing no form of barrier protection from attack of the corrosive species in the salt water.

Stability of corrosion products on the surface of the metal could play a key role in the corrosion inhibition of Neem leaf extract. When the corrosion of metal is accompanied by the formation of a protective layer of corrosion products, the corrosion rate is determined by one of the following stages; the diffusion of the oxidant to the metal surface through the corrosion product layer; the corrosion reaction rate; or the diffusion rate of the corrosion products from the metal surface towards the bulk solution. The slowest step determines the metal corrosion rate [16].

\section{CONCLUSION}

Mild steel samples immersed in salt water without inhibitor exhibited a much higher weight loss in all test samples as compared to those in the salt water with Neem leaf extract as inhibitor. Weight loss of as-received samples was higher than heat treated prior to the addition of inhibitor into the corrosive medium. Reverse was the case on the addition of Neem leaf extract as inhibitor. Generally, the results show that Neem leaf extract is a good corrosion inhibitor of mild steel in salt water environment. Also, corrosion rate was least with 5\% inhibitor and increased with increase in inhibitor concentration for both as-received and heat treated coupon. Although, 5\% inhibitor appears to be the optimum inhibitor concentration for mild steel corrosion in the present study, investigation of lower concentrations may be necessary. Also because laboratory immersion test does not consider conditions where agitation and flow may be required, it is recommended that in conditions where wave motion and temperature fluctuations come into play, these should be considered as they could negatively affect inhibitor adsorption.

\section{ACKNOWLEDGEMENTS}

The authors would like to thank Bonny Eneye for his hard work during this project.

\section{REFERENCES}

[1] I.A. Zaafarany, Corrosion Inhibition of Mild Steel in Hydrochloric Acid Solution using Cationic Surfactant Olyel-amido Derivatives Int. J. Electrochem. Sci.,Vol. 8, pp 9531 - 9542 (2013)

[2] Richard Guise, www.finishing.com/124/97.shtml

[3] Clowesoft, www.finishing.com/133/90.shtml

[4] P. B. Raja and M. G. Sethuraman, Materials Letters Vol. 62, pp 2977-2979 (2008)

[5] L.R Chauhan and G. Gunasekara, Corrosion inhibition of mild steel by plant extract in dilute $\mathrm{HCl}$ medium, Corrosion Science Vol. 4, pp. 1143-1161, (2007)

[6] M. Lebrini, F. Robert and C. Roos, Alkaloid extract from paliocourea Guianensis plant as corrosion inhibitor for C38 steel in !M Hydrochloric acid medium, Int. J. Electrochem. Sci., Vol. 6, pp. 847-859 (2011).

[7] R. Saratha and V. G. Vasudha, Emblica Officinalis (Indian Gooseberry) leaves extract as corrosion inhibitor for mild steel in $1 \mathrm{~N} \mathrm{HCl}$ medium. E-journal of Chemistry, Vol. 7(3), pp. 677-684 (2010).

[8] M. Abde-Gaber, B. A. Abd-El-Nabay, I. M. Sidahmed, A. M. El-Zayady, M. Saadawy, Kinetics and thermodynamics of aluminium dissolution in 1.0M Sulphuric acid containing chloride ions, Corr. Sci. Vol. 48, pp 2765-2779, (2008).

[9] Nahle, I. Abu-Abdoun, I. Abdel-Rahman and M. AlKhayat, UAE Neem extract as a corrosion inhibitor for carbon steel in $\mathrm{HCl}$ solution. International Journal of Corrosion, Vol. 2010, Article ID 460154, 9 pages, doi: $10.1155 / 2010 / 460154$

[10] V. S. Sastri, Corrosion Inhibitors, Principles and applications, pp. 45 (2001)

[11] O. P. Khanna, A Text Book of Materials Science and Metallurgy, pp. 43-1, (2007)

[12] H. M. Abd El-Lateef, V. M. Abbasov, L. I. Allyeva, T. A. Ismayilov, Corrosion protection of Steel 
Pipelines Against $\mathrm{CO}_{2}$ Corrosion-A Review, Chemistry Journal, Vol. 02, pp.52-63 (2012).

[13] E. Naderi, A.H. Jafari, M. Ehteshamzadeh, M.G. Hosseini, Effect of carbon steel microstructures and molecular structure of two new Schiff base compounds on inhibition performance in $1 \mathrm{M} \mathrm{HCl}$ solution by EIS, Materials Chemistry and Physics; Vol. 115 pp 852-858 (2009).

[14] S. Hernandez, J. Bruzual, F. Lopez-Linaries and J. Luzon; Isolation of potential Corrosion Inhibiting Compounds in Crude Oil.; Proceedings of CORROSION/2003. NACE International, Houston, Texas, Paper no. 2293.

[15] R. E. Smallman and A. H.W. Ngan, Physical Metallurgy and Advance Materials, Seventh Edition, pp. 421-422 (2007)

[16] Z. Panossian, N. Lira de Almeida, R. M. Ferreira de Sousa, Gutemberg de Souza Pimenta, L. B. Schmidt Marques, Corrosion of carbon steel pipes and tanks by concentrated sulphuric acid: A review; Corrosion Science Vol. 58, pp. 1-11 (2012) 